\title{
Decomposed Algorithm for Risk-Constrained AC OPF with Corrective Control by Series FACTS Devices
}

\author{
Dmitry Shchetinin*, Gabriela Hug \\ ETH Zurich \\ Physikstrasse 3, Zurich, Switzerland
}

\begin{abstract}
Maintaining a desired level of security in a power system while maximizing its efficiency is a cornerstone of system operation. In recent years, system security has been jeopardized by increasingly variable power flow patterns caused by renewable generation and market liberalization. One potential solution is to employ corrective power flow control by means of series FACTS devices. However, this requires solving a complex optimization problem, which is difficult to do quickly for large-scale power systems. While several decomposition strategies have been proposed to address this issue, existing approaches rely on a DC approximation of a power system model and thus do not fully capture system's behavior. This paper presents a decomposed iterative algorithm for the probabilistic security-constrained OPF problem, which is formulated for an AC model of a power grid with series FACTS devices and outages of more than one element as credible contingencies. The algorithm is based on so-called locational security impact factors, which represent sensitivities of the security index to changes in generator outputs. The IEEE 24-bus and 118-bus systems are used to examine the effectiveness of the proposed method and to analyze the effect that series FACTS devices have on security and efficiency of system operation.
\end{abstract}

Keywords: optimization, power system security, flexible AC transmission systems, risk-based optimal power flow, corrective control

\section{Introduction}

With the liberalization of electricity markets and higher penetration of renewable generation the problem of balancing security and efficiency of power system operation is becoming increasingly challenging. Excessively rigorous se5 curity criteria may lead to a sub-optimal generation dispatch, reducing social

\footnotetext{
Funding: This work was supported by the National Science Foundation [grant number ECCS-1252944] and internal funds of ETH Zurich.

* Corresponding author

Email addresses: shchetinin@eeh.ee.ethz.ch (Dmitry Shchetinin), hug@eeh.ee.ethz.ch (Gabriela Hug)
}

Preprint submitted to Electric Power Research Journal

July 11, 2016 
welfare, whereas sacrificing security for efficiency may result in major blackouts. Hence, quantifying security of power systems and enhancing it while keeping the total supply cost as low as possible are important aspects of system operation.

Traditionally, system security has been quantified by deterministic approaches such as the N-1 criterion [1, 2, 3. Despite its simplicity, this criterion treats all outages equally in terms of both their probability to occur and their effect on the system, which does not reflect reality and may lead to inefficient operation. To overcome this drawback, probabilistic approaches to system security, based on a notion of risk [4, 5, 6, 7, have been proposed. Risk-based security indices 15 can be used for both increasing operators' awareness of the system state [8, 9] and optimizing power system performance 10, 11, 12, e.g. by formulating the Risk-Constrained OPF (RCOPF) problem [13, 14, 15, 16, 17, 18.

Power system security can be enhanced by generation re-dispatch 2, load shedding 19, 20, or power flow control [21, 22, e.g. by means of FACTS devices

20 23. The latter is preferable as its cost is limited to the procurement and maintenance cost of control devices, whereas the other two reduce social welfare and should be ideally avoided. Since FACTS devices are fast-acting, they enable the implementation of corrective power flow control [24, i.e. the set-points of the devices can be changed quickly according to the state of the system. While

25 FACTS devices are not widely used due to their high cost, recent developments such as Distributed FACTS [25] are promising as they offer potentially reduced installation and maintenance costs, which can lead to their extensive use.

Both the deterministic and probabilistic security-constrained OPF problems are notorious for their difficulty because each is a large-scale, nonlinear, non30 convex optimization problem that usually has to be solved as quickly as possible. Due to its practical importance, the security-constrained OPF problem has received a lot of attention from the research community and a number of formulations and solution algorithms can be found in the literature. An extensive overview of major challenges and existing endeavors in this field has been presented in [26] and, more recently, [27.

This paper focuses on the RCOPF problem, which has the objective of minimizing the total supply cost in the system while keeping the risk below a given value and, due to its size, becomes difficult to solve in a centralized fashion for real-world grids. Existing approaches for decoupling this problem include 40 using Benders decomposition [14] or so-called locational security impact factors (LSIFs) [15, 28, 29]. However, studies [14, 15, 28] are based on a DC approximation of a power system, and the problem formulation in [29] does not include corrective control. In addition, only single-element outages are considered in these studies and communication overhead between parallel processors is not 45 taken into account when analyzing the potential acceleration of the algorithm with parallelized computations.

The main contributions of this paper are threefold. First, it presents a decomposed iterative algorithm for solving the RCOPF problem for a full AC model of a power system with corrective control. The utilization of the AC ${ }_{50}$ model poses certain challenges, e.g. some contingencies may have no steadystate solution. The paper discusses a possible strategy of dealing with such 
contingencies. Second, the paper considers outages of more than a single element in the system, which might help capture potential danger posed by cascading outages and provide system operators with a better risk estimate. For the sake of presentation simplicity, the paper is focused only on N-1 and N-2 outages, but the proposed algorithm can be straightforwardly extended to include any N-k outages as credible contingencies. The last focus of the paper is to examine what the best way of parallelizing the proposed algorithm is and how communication overhead influences the simulation time. In addition, the paper analyses the

60 impact that series FACTS devices have on the risk and cost of system operation as well as on the computation time.

The rest of the paper is structured as follows: Sections 2 and 3 provide an overview of steady-state models of series FACTS devices and risk-based security indices, respectively. In Section 4 locational security impact factors are 65 described. In Section 5, the centralized formulation of the AC RCOPF is presented and Section 6 provides the description of the proposed iterative algorithm for solving the AC RCOPF problem. In Section 7, simulation results are provided, and Section 8 concludes the paper.

\section{Series FACTS Modeling and Control}

In general, security of a power system depends on both voltage magnitudes at buses and currents in transmission lines and transformers. However, in this paper only the effect of the line flows on reliability is taken into account, which is why series FACTS devices are considered as a means of power flow control. Examples of such devices include the Thyristor Controlled Series Compensator

75 (TCSC), Static Synchronous Series Compensator (SSSC) [12, and recently developed distributed FACTS (DFACTS) [25] devices. For steady-state analysis, all aforementioned devices can be modeled as a variable reactance inserted in series into the transmission line. By changing the value of $X_{F A C T S}$, the susceptance of a transmission line with a series FACTS device can be changed, which

so in turn causes the change in the line flow, thus enabling power flow control. All power electronics-based FACTS devices are fast-acting, which provides an opportunity of quickly adjusting line flows after the occurrence of a disturbance to eliminate possible violations of operational constraints. This may allow the operator to choose a generation dispatch with a lower total supply cost while

85 still satisfying security requirements. Such a control strategy, which is called corrective power flow control, is considered in this paper.

\section{Risk-Based Security Indices}

As was mentioned in the introduction, probabilistic approaches to system security are based on a concept of risk, which for a certain event is defined as

9o the product of its probability to occur and the consequences that will ensue. The events are represented by outages of various elements in the system called contingencies and the total risk is the sum of individual risks of all considered 
contingencies along with the normal state. The value of the total risk provides a quantitative measure of system security, as opposed to a binary N-1 criterion.

95 The risk value can be used as a constraint in OPF to determine the most costefficient generation dispatch that still satisfies security requirements.

Unfortunately, at this point there is no benchmark method of selecting the contingencies to be included in the risk calculation as well as quantifying the consequences of considered contingencies. Proposed risk metrics vary substanthat this paper does not aim at developing a new risk index and comparing this index with others found in literature. Rather, it adopts already existing metrics that are deemed well suited for the main focus of this study, which is the real-time optimization of power system operation.

Existing approaches to quantifying the consequences of contingencies can be divided into two groups. The first group determines the economic effect of contingencies [5, 12] using various indices such as expected energy not served (EENS), loss of load probability (LOLP), etc. This approach assumes certain actions from a system operator and its main application is in medium and 110 long-term planning. The second group [9, 4] uses a purely technical definition of consequences as functions of the loadings of system elements. Here, the risk index is independent of any assumptions regarding the actions of a system operator, but rather reflects the level of danger to the system if no such actions are undertaken. This approach is well suited for short-term planning or system operation and, therefore, it is employed in this paper.

Contingencies for the risk calculation can be chosen either by the MonteCarlo approach [5] or from a pre-determined list of outages that are deemed credible 4. In this paper, the latter approach is employed with considering all N-1 and N-2 outages to be credible. This is done in order to capture the risk pertaining to cascading outages, which may occur if the outage of a single element leads to high loadings of other elements, making them more likely to become disconnected and cause further exacerbation of the system state.

To compute the risk of a certain contingency, the following procedure is used. First, the operational constraints such as line flow limits are relaxed by adding slack variables:

$$
h_{p, q}\left(P_{G}, x_{q}\right) \leq k_{p} \cdot \bar{h}_{p}+s_{p, q}, \quad s_{p, q} \geq 0
$$

where $h_{p, q}$ is the operational constraint of element $p$ in contingency $q$, which is a function of generator outputs $P_{G}$ and control variables $x_{q}, \bar{h}_{p}$ is the limit on this constraint, and $s_{p, q}$ is the corresponding slack variable. The slack variable represents the part of the loading of the corresponding element that is considered to be unsafe. Coefficient $0<k_{p} \leq 1$ controls the loading level at which the slack variable becomes non-zero and starts to contribute to the risk index.

To quantify the consequences of a contingency, so-called severity functions introduced in [9] are used. For a certain element, the severity function, shown in Fig. 1a is a quadratic function of its loading. The consequences value for a given system state is determined as the sum of the severity functions values for 


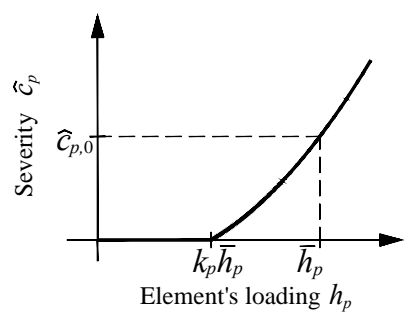

(a) Severity function

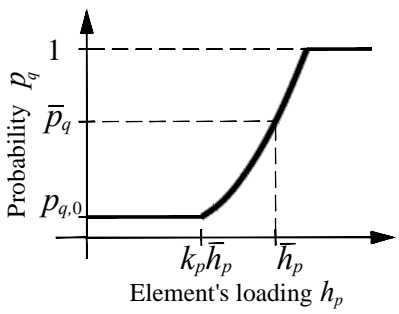

(b) Probability function

Figure 1: Probability and severity functions

all elements that are still in operation in this state:

$$
c_{q}=\sum \hat{c}_{p}\left(s_{p, q}\right)
$$

The probability of element $p$ to fail and create contingency $q$, shown in Fig. 1b is chosen based on 15 to have two components. The first is constant and represents the outage probability obtained from historical records, and the second is a quadratic function of the element's loading before the outage. This allows capturing a cascading effect for contingencies with two outages, where the probability of a second element to fail is affected by the system state after 135 the failure of a first element. Hence, this is based on the assumption that independent simultaneous outages of two elements are extremely unlikely.

The total risk of system operation is simply the sum of the risks of all considered system states:

$$
r_{\text {tot }}=\sum_{q=0}^{S} p_{q} \cdot c_{q}
$$

where $S$ is the total number of considered contingencies and $q=0$ corresponds to the normal state.

\section{Locational Security Impact Factors}

Locational security Impact Factors (LSIFs), which represent the sensitivities of the total risk in the system to the changes in active power outputs of generators, were first introduced in 28] for a DC approximation of a power system and in [29, this concept was extended to a full AC model. LSIFs can provide useful information to system operators as they show what generators to re-dispatch in

145 order to reduce the risk of system operation. In addition, these sensitivities can be used to decompose risk-constrained OPF.

Previously, LSIFs were applied to the risk formulation in which considered contingencies included only the outages of single elements in the system. While the nature of LSIFs does not depend on what contingencies are deemed credible, extending the risk formulation to include $\mathrm{N}-2$ level contingencies requires a new 
computational algorithm. In case that all $\mathrm{N}-1$ and $\mathrm{N}-2$ contingencies along with the normal state are considered, (3) can be reformulated as

$$
r_{\text {tot }}=c_{0}+\sum_{q=1}^{N} p_{q} \cdot \underbrace{\left(c_{q}+\sum_{v=1, v \neq q}^{N} p_{v \mid q} \cdot c_{v \mid q}\right)}_{c_{q}^{\text {tot }}}
$$

where $c_{0}$ corresponds to the consequences value for the normal system state, $N$ is the number of first-level contingencies, $p_{q}$ and $c_{q}$ are the probability and consequences of a first-level contingency, and $p_{v \mid q}$ and $c_{v \mid q}$ are the probability and consequences of second-level contingency $v$ provided that first-level contingency $q$ has occurred. Thus, $c_{q}^{t o t}$ represents the consequences of the first-level contingency that also include the total risk of all second-level contingencies associated with this first-level contingency.

The sensitivity of the total risk with respect to the output of generator $i$ is:

$$
\begin{gathered}
s_{i}=\frac{\partial r_{t o t}}{\partial P_{i}}=\frac{\partial c_{0}}{\partial P_{i}}+\sum_{q=1}^{N} \frac{\partial p_{q}}{\partial P_{i}} \cdot\left(c_{q}+\sum_{v=1, v \neq q}^{N} p_{v \mid q} \cdot c_{v \mid q}\right)+ \\
\sum_{q=1}^{N} p_{q} \cdot\left(\frac{\partial c_{q}}{\partial P_{i}}+\sum_{v=1, v \neq q}^{N} c_{v \mid q} \cdot \frac{\partial p_{v \mid q}}{\partial P_{i}}+\sum_{v=1, v \neq q}^{N} p_{v \mid q} \cdot \frac{\partial c_{v \mid q}}{\partial P_{i}}\right)
\end{gathered}
$$

For generator $i$, LSIF $s_{i}$ shows how much the total value of risk would be affected by a change in the output of generator $i$ and the opposite corresponding change in the output of the slack bus 28 . Therefore, it is the difference between the LSIFs of two generators that determines the impact of the re-dispatch of their active power outputs on the total risk value rather than the absolute values of the LSIFs themselves.

The method of calculating LSIFs and their application to decomposing the $\mathrm{RCOPF}$ problem are explained in Sec. 6 of this paper.

\section{Centralized Algorithm for AC RCOPF with Corrective Control}

The purpose of RCOPF is to determine the generation dispatch with the minimum possible total supply cost while ensuring that the risk is kept below a pre-defined value. If fast-acting FACTS devices are installed in the system, corrective power flow control can be executed, i.e. the settings of FACTS devices for every system state are selected such that the contribution of this state to the total risk is minimized for a given generation dispatch. Mathematically, this results in the following optimization problem:

$$
\begin{gathered}
\min \sum_{l=1}^{N_{G}} C_{l}\left(P_{G_{l}}\right) \\
W_{P_{i}, q}=0, \quad i=1, \ldots, N_{B}
\end{gathered}
$$




$$
\begin{array}{lr}
W_{Q_{i}, q}=0, & i=1, \ldots, N_{P Q} \\
P_{G_{l}}^{\text {min }} \leq P_{G_{l}, q} \leq P_{G_{l}}^{\max }, & l=1, \ldots, N_{G} \\
V_{i}^{\text {min }} \leq V_{i, q} \leq V_{i}^{\max }, & l=1, \ldots, N_{B} \\
I_{i j, q} \leq I_{i j}^{*}+s_{i j, q}, & i j \in \Omega_{L} \\
X_{i j, \text { min }}^{F A C T S} \leq X_{i j, q}^{F A C T S} \leq X_{i j, \max }^{F A C T S}, & i j \in \Omega_{T} \\
r_{\text {tot }} \leq r_{\max } &
\end{array}
$$

$$
\text { all for } q=0, \ldots, S
$$

where $q$ indicates a particular system state, $N_{G}, N_{B}, N_{P Q}$ are the total number of generators, buses, and PQ-buses, respectively, $P_{G_{l}}$ is the active power output 165 of generator $l$ and $C_{l}\left(P_{G_{l}}\right)$ is its cost function, $\Omega_{L}$ is the list of the branches, $\Omega_{T}$ is the list of all series FACTS devices, $W_{P_{i}}$ and $W_{Q_{i}}$ are active and reactive power balance equations for node $i, I_{i j}$ and $I_{i j}^{*}=k_{i j} I_{i j}^{\max }$ are the current through line $i j$ and its reduced limit as in (1), respectively, $s_{i j, q}$ is the corresponding slack variable representing unsafe part of the line's loading, the lower and upper 170 limits on variables are denoted by superscripts $\min$ and $\max , r_{\text {tot }}$ is the total risk as defined in (4) and $r_{\max }$ is the maximum allowable level of risk. Note that the active power balance for the slack bus should be included only for the normal state, because in all contingencies the generation at the slack bus varies to allow for changed active power losses.

Although the formulation of this optimization problem is rather straightforward, its computational complexity is substantial. Since N-1 and N-2 contingencies are deemed credible, the total number of variables and constraints is proportional to $L^{3}$, where $L$ is the number of transmission lines and transformers in the system. While generator outages also constitute danger to system security, only branch outages are considered to be credible contingencies in this paper to limit its scope. It is known that the most demanding operation in large-scale optimization is the repeated solution of the linear system. The complexity of this operation grows nonlinearly with the system size and for an $n \times n$ matrix can be roughly described as $\mathcal{O}\left(n^{a}\right)$ with $a>2$. Therefore, for large-scale 185 power systems the centralized solution algorithm is computationally inefficient and other methods have to be sought to enable the application of RCOPF in control centers. Another drawback of this method is that if there is no power flow solution to any of considered contingencies, the feasible solution to the optimization problem does not exist. 

in generator outputs. A detailed description of each step of the algorithm is provided below. 
Step 0. Unconstrained OPF: First, a generation dispatch that corresponds to the minimum possible total supply cost while satisfying the power balance equations is determined by solving the optimization problem given by (6)-(8). This is the minimum possible supply cost the system can have for a given load pattern. Note that this step does not yet include any security constraints.

Step 1. Second-level Consequences Calculation: Once the initial dispatch has been determined, the consequences for all contingencies with two outages are calculated for this dispatch. Note that at this point the order in which two elements are disconnected from the grid does not matter since the resulting system state is the same. To determine the minimum possible consequences value $c_{v \mid q}$ for second-level contingency $v$ caused by first-level contingency $q$, the following optimization problem is formulated and solved:

$$
\min _{X_{F A C T S, v \mid q}} c_{v \mid q}
$$

s.t. (7)-(8) and (10)- 13 )

At the optimum solution, the Lagrange Multiplier value for the active power balance constraint for generator $i$ corresponds to:

$$
\lambda_{i, v \mid q}=\frac{\partial c_{v \mid q}}{\partial P_{i}}
$$

The optimum values for consequences $\bar{c}_{v}$ along with Lagrange Multipliers $\boldsymbol{\lambda}_{v \mid q}$ are recorded and the algorithm proceeds to the next step.

Step 2. First-level Consequences Calculation: After the consequences for all considered N-2 level contingencies have been determined, the same is done for all considered N-1 level contingencies with adding the risk of all corresponding second-level contingencies. Thus, for contingency $q$ the following optimization problem is formulated and solved:

$$
\min _{X_{F A C T S, q}}\left(c_{q}+\sum_{v=1, v \neq q}^{N} p_{v \mid q} \cdot \bar{c}_{v \mid q}\right)
$$

s.t. (7)-(8) and (10)-(13)

At the optimum solution, the Lagrange Multiplier value for the active power balance constraint for generator $i$ corresponds to:

$$
\lambda_{i, q}=\frac{\partial c_{q}}{\partial P_{i}}+\sum_{v=1, v \neq q}^{N}\left(\frac{\partial p_{v \mid q}}{\partial P_{i}} \cdot \bar{c}_{v \mid q}\right)
$$

Before proceeding to the next step, the optimized values for consequences $\bar{c}_{q}^{\text {tot }}$ along with Lagrange Multipliers $\boldsymbol{\lambda}_{q}$ and probabilities of second-level contingencies $p_{v \mid q}$ given the occurrence of first-level contingency $q$ are recorded.

Step 3. Total Risk and LSIFs Calculation: Finally, the total risk of system 
operation is determined:

$$
\min _{X_{F A C T S, 0}}\left(c_{0}+\sum_{q=1}^{N}\left(p_{q} \cdot \bar{c}_{q}^{t o t}\right)\right)
$$

s.t. $(7)-(8)$ and $(10)-(13)$

At the optimum solution, the Lagrange Multiplier value for the active power balance constraint for generator $i$ corresponds to:

$$
\lambda_{i, 0}=\frac{\partial c_{0}}{\partial P_{i}}+\sum_{q=1}^{N}\left(\frac{\partial p_{q}}{\partial P_{i}} \cdot \bar{c}_{q}^{t o t}\right)
$$

If the value of the total risk is below the given limit, the algorithm stops. Otherwise, the LSIFs defined in (5) for the current generation dispatch are determined using the obtained Lagrange Multipliers according to:

$$
s_{i}=\lambda_{i, 0}+\sum_{q=1}^{N} \lambda_{i, q} \cdot p_{q}+\sum_{q=1}^{N}\left(p_{q} \cdot \sum_{v=1, v \neq q}^{N} \lambda_{i, v} \cdot p_{v}\right)
$$

These values are used in the risk-cost trade-off step, which is described below.

Step 4. Risk-Cost Trade-Off In order to obtain the most cost-efficient redispatch while taking into account the information provided by LSIFs, the cost minimization OPF problem given by (6)-(8) is solved once again with an additional LSIF-based constraint:

$$
r_{\text {tot }}^{(j)}+\sum_{l=2}^{N_{G}}\left(s_{l}^{(j)} \cdot\left(P_{G_{l}}-P_{G_{l}}^{(j)}\right)\right) \leq r_{\max }, \quad j=1, \ldots, k
$$

where $j$ is the index of the current iteration and the slack bus is assumed to have index 1. Thus, at each iteration one more LSIF-based constraint is added to the constraint set and the algorithm proceeds to Step 1. These constraints contain the information regarding how to re-dispatch the generation in order to reduce the total risk value. Since they are based on the linearization of the non-linear system, several iterations are required to reduce the inaccuracies introduced by such a linearization to an acceptable level. The stopping criteria may include the satisfaction of the risk constraint, reaching a certain pre-specified number of iterations, or the change in the generation dispatch on two consecutive iterations being smaller than a given tolerance.

255 Such an iterative algorithm can significantly reduce the computation time compared to the centralized method. The first reason is that the computation time of the iterative algorithm grows linearly with the number of considered system states. The second reason is that optimization subproblems for contingencies can be solved in parallel, which is examined later in this section. 


\subsection{Handling Contingencies with no Power Flow Solution}

If the AC model of a power system is utilized, it is possible that for some contingencies there exists no power flow solution, which physically means that generated power cannot be transmitted through the grid. It is worth noting that while theoretically this may be the case for any system state, for a realistic grid in most cases this problem most likely would only arise after unexpected outages of at least two elements. When solving the RCOPF problem in a centralized way, even one such contingency will lead to the inability of the algorithm to converge. The only way to ensure that all contingencies result in stable operating points in case the re-dispatch alone is not sufficient is to perform load shedding. However, it would be difficult to incorporate the load shedding strategy into the centralized RCOPF problem as it would require weighting the cost of shedding against the supply cost. Furthermore, the risk value in this case would be computed with an assumption of certain actions of the system operator, which is undesirable.

In the iterative approach, every system state is considered separately, which provides more flexibility for dealing with contingencies with no power flow solution. In this paper, the following strategy is proposed. Once such a contingency has been detected, it is excluded from the risk calculation, since no meaningful value of consequences can be obtained in this case. The outages that lead to this contingency are recorded and can be viewed by a system operator. Since such a contingency is most likely an N-2 contingency and, therefore, has a very low probability to occur, the optimal generation dispatch can be computed disregarding this contingency. However, it might be of interest for the system operator how much re-dispatch and/or load shedding would be required, should

285 this contingency happen. Therefore, for each contingency with no power flow solution an optimization problem can be formulated, which has the objective of minimizing the combined cost of re-dispatch and load-shedding required to ensure that this system state is feasible and/or does not violate additional security criteria such as line flows, bus voltages etc. Thus, the iterative approach can provide the operator with the list of all infeasible system states and the minimum cost of eliminating each of these infeasibilities while leaving the risk calculation independent of any assumptions regarding the possible actions that the operator might undertake.

\subsection{Parallelization of Iterative Algorithm}

An important advantage of the proposed iterative algorithm over the centralized one is that at each iteration consequences of contingencies can be computed in parallel, thus further reducing the computation time. The scheme of each iteration is shown in Fig. 2, where each rectangle represents an optimization subproblem. The iteration starts with obtaining a certain generation dispatch. Then the consequences for all N-2 contingencies are determined, followed by calculating the consequences of $\mathrm{N}-1$ contingencies, and, finally, the total risk. Two things are important to note here. The first is that the consequences of an N-1 contingency can be obtained only after the consequences of all $\mathrm{N}-2$ contingencies 
'inside' this contingency have been computed. Second, this paper assumes that the order in which two elements are disconnected does not affect the resulting system state and the corresponding consequences value, which makes it possible to compute the consequences for only half of the considered N-2 contingencies.

While it seems natural to directly parallelize the loop going over all $\mathrm{N}-2$ contingencies, such strategy would result in a significant communication overhead 310 between the parallel workers and the client. On the other hand, parallelizing the loop going over N-1 contingencies would require each parallel worker to compute the consequences of all corresponding N-2 contingencies. Therefore, the fact that in total only half of the N-2 contingencies has to be computed would not be utilized.

For the aforementioned reasons, the following parallelization scheme is proposed in the paper. First, each parallel worker is given an N-1 contingency with those corresponding N-2 contingencies that are not sent to other workers. The worker computes the consequences of all N-2 contingencies and sends the result to the client in one block. After this step, the consequences of all N-2

320 contingencies are known. Next, a parallel loop going over all N-1 contingencies is organized, but this time each worker only has to compute the consequences of the corresponding N-1 contingency. Once the consequences of all N-1 contingencies have been obtained, the client is able to calculate to total risk value. Such a scheme enables reduced communication overhead while including only half of the N-2 contingencies in the computations.

\section{Simulations}

This section presents and discusses simulation results that illustrate the performance of the proposed iterative algorithm and the impact of series FACTS devices on the risk and cost of system operation.

\subsection{Simulation Setup}

The IEEE 24-bus [30] and 118-bus systems were used in this study. Supply costs were chosen to be quadratic functions of the corresponding active power

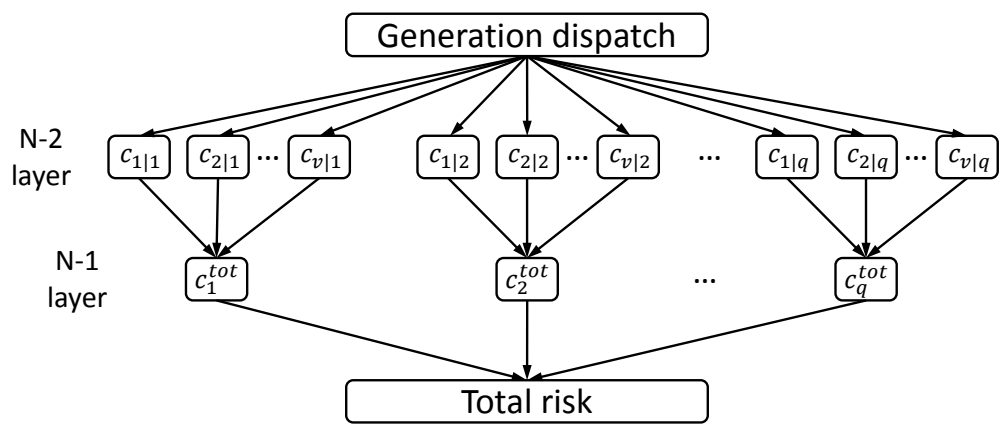

Figure 2: Flowchart of one iteration of the iterative algorithm 
outputs:

$$
C\left(P_{G_{i}}\right)=a_{i} \cdot P_{G_{i}}^{2}+b_{i} \cdot P_{G_{i}}+c_{i}
$$

The 24-bus system was used for the comparison of the centralized and iterative methods for solving the AC RCOPF and for all other simulations the 118-bus system was used. The summaries for both systems are provided in Table 1 . which also contains the number of considered N-1 and N-2 outages, the total number of system states as well as the total number of constraints and variables (without and with FACTS devices) in the centralized RCOPF.

In order to analyze the effect of corrective power flow control on system performance, series FACTS devices were installed on twelve lines in the 24-bus system and twenty five lines in the 118-bus system. Each device was chosen to have the regulation range of $50 \%$ of the corresponding line's reactance both in capacitive and inductive modes. Currently, these devices are expensive and only a few of them are installed in real power grids. However, recent developments such as DFACTS [25] indicate that the prices for power flow control devices may decrease in the future, which could lead to their extensive use. Thus, this 345 paper investigates the impact that a high penetration of such devices would have on the risk and cost of system operation as well as on the complexity of the corresponding optimization problem.

The list of chosen contingencies included all combinations of outages of up to two lines/transformers that did not lead to islanding of any nodes. To limit the scope of this paper, other types of contingencies were not considered.

Coefficient $k_{p}$ was chosen to be equal to 0.7 , which means that an element starts to contribute to the consequences value once its loading exceeds $70 \%$ of nominal. The severity function was designed such that its value is equal to 0.5 at the operational limit of a line/transformer and 1 at $120 \%$ loading. For simplicity, the constant term in the probability function $p_{q, 0}$, which reflects the statistical outage probability, was chosen to have a value of 0.003 for all outages and the probability of the normal state was assumed to be 1 . The parameters of the probability function were selected in such a way that an element has the outage probability of 0.5 at $100 \%$ loading and 1 at a $20 \%$ overload.

360 Apart from the base case, nine different configurations of each system were developed. These configurations were obtained by randomly changing the loading pattern based on a normal distribution in order to simulate different operating conditions. Hence, a total of twenty system configurations were considered for each system, ten with FACTS devices and ten without FACTS devices.

Unless stated otherwise, all simulations were carried out on a $2.9 \mathrm{GHz}$ fourcore $\mathrm{PC}$ with $32 \mathrm{~Gb}$ of RAM. All optimization problems were solved using an

Table 1: Parameters of 24-bus and 118-bus systems

\begin{tabular}{|c|c|c|c|c|c|c|c|c|}
\hline Nodes & Lines & Gens & FACTS & N-1 & N-2 & States & Cons & Variables \\
\hline 24 & 38 & 33 & 12 & 37 & 656 & 694 & 50,009 & $58,350 / 66,527$ \\
\hline 118 & 186 & 54 & 25 & 177 & 15,502 & 15,680 & $5,723,381$ & $6,585,833 / 6,616,841$ \\
\hline
\end{tabular}


optimization package called TOMLAB through a MATLAB interface. For parallelized parts of the iterative algorithm, four parallel workers were used.

Note that it is not the purpose of this paper to determine suitable values for the probability and severity functions as well as the acceptable value of the total risk. It is assumed that system operators will be able to provide these values based on their experience and knowledge of their systems. The focus of this section is to study different aspects of the proposed algorithm and to analyze the impact that corrective power flow control has on the system performance.

\subsection{Iterative Algorithm Performance}

To study the performance of the iterative approach, the 118-bus system with and without FACTS devices was used. First, for each system configuration, the minimum possible supply cost was determined in the absence of any security requirements. This was done by solving the AC OPF problem of Step 0 of the 380 iterative algorithm. Next, the corresponding total risk value for the obtained dispatch was calculated. Then, the maximum allowable risk level was set to $30 \%$ of the computed initial risk value. To obtain the most cost-efficient generation dispatch that would satisfy this risk constraint, the AC RCOPF problem was solved by the proposed iterative algorithm. The algorithm was considered to have converged if the violation of the risk constraint becomes smaller than $0.001 \%$. The evolutions of the total risk and cost are shown in Fig. 3, where each line corresponds to one system configuration. The risk and cost are presented in p.u., with the initial values of the risk and cost being their base values. As can be seen from the figure, in order to satisfy the risk constraint the total 390 supply cost increased due to generation re-dispatch.

A number of observations can be made from the obtained simulation results. First, the algorithm had a similar convergence pattern both for the cases with and without TCSCs, which shows that not only was it able to handle a nonlinear AC problem, but it also coped with additional non-linearities introduced by the presence of multiple series FACTS devices in the system. Second, while on average the algorithm needed from 13 to 20 iterations to converge with an average of 17 iterations, in all but one case the violation of the risk constraint was already less than $1 \%$ after ten iterations. Therefore, by varying the set tolerance of the risk constraint violation it is possible to significantly affect the 400 algorithm's computation time. Additional experiments showed that the smaller the required reduction in the total risk was, the faster the algorithm converged. When the desired risk was set to $50 \%$ and $70 \%$ of the initial value, the average number of iterations reduced to 13 and 8 , respectively.

Thus, the iterative algorithm demonstrated robust convergence for all test cases regardless of the risk limit and presence of FACTS devices in the system.

\subsection{Centralized vs Iterative Approach}

In this section, the results obtained by the iterative algorithm were compared to the centralized solution in terms of the solution accuracy and simulation time. Since for the 118-bus system the total number of variables was approximately 6.6 


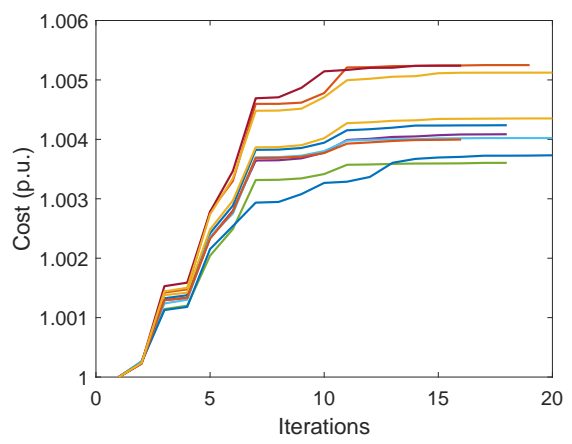

(a) Cost evolution without FACTS

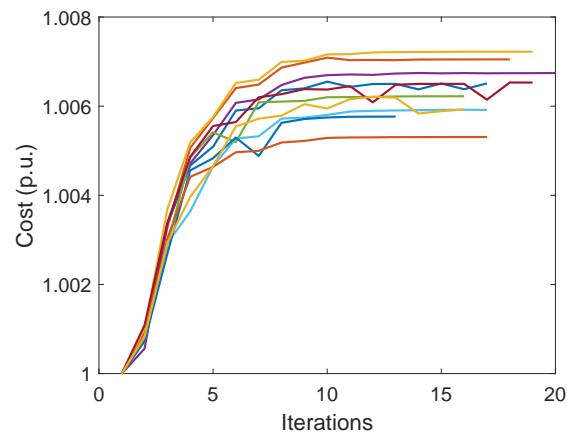

(c) Cost evolution with FACTS

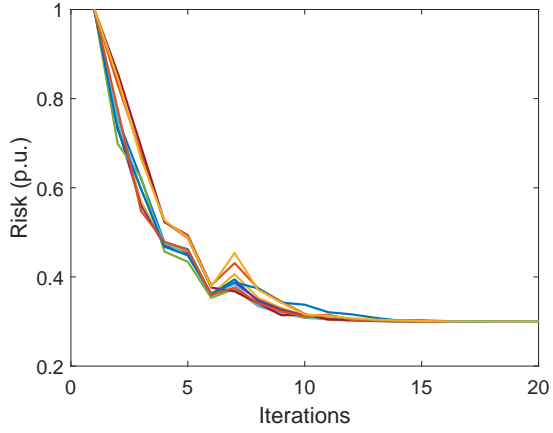

(b) Risk evolution without FACTS

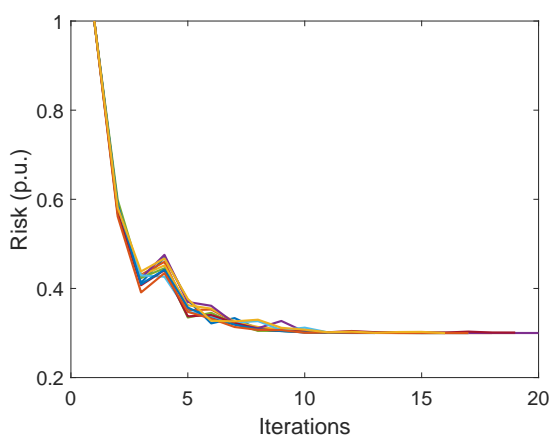

(d) Risk evolution with FACTS

Figure 3: Risk and cost evolution for system with and without FACTS

million, the resulting optimization problem could not be solved in a centralized manner in reasonable time. For this reason, the 24-bus system was used to compare the two approaches.

For each test case, the maximum allowable risk level was set to $30 \%$ of the initial risk value corresponding to the most cost-efficient generation dispatch. As was explained in Sec.6.2, the centralized algorithm fails to converge if any of the considered contingencies does not have a steady-state solution for a given loading pattern. Therefore, the AC RCOPF problem was first solved by the iterative approach, which along with the resulting generation dispatch provided the list of all such contingencies. After that, the centralized algorithm was employed with 420 the obtained contingencies excluded from the list of considered system states. Table 2 shows the comparison results for all ten system configurations without series FACTS devices. In the table, 'flat' and 'warm' refer to the starting values of variables: 'flat' means that a flat start was used with voltage magnitudes equal to 1 p.u. and phase angles equal to zero, and 'warm' indicates that the initial values were obtained from the solution of the regular AC power flow for the initial generation dispatch. 
As can be seen from the table, the total supply cost, obtained by the iterative algorithm, was very close to the centralized one, which means that both algorithms converged to the same solution. However, the iterative approach was on average 33 times faster in case of the 'warm start' and 166 times faster in case of the 'flat start'. Thus, even for a small test system, the iterative algorithm is significantly faster and less sensitive to the starting point than the centralized one. Note that the results for the iterative algorithm were obtained using only four parallel workers. Adding more parallel processors would have reduced the 435 computation time of the iterative algorithm even further, which is discussed in more detail in Sec. 7.5 .

When series FACTS devices are included in the optimization, a lot of independent control variables are added into the problem, which significantly increases the complexity of the centralized RCOPF problem. With the computational power at the authors' disposal, it was not possible to carry out simulations for all system configurations in reasonable time. Therefore, only the base case was considered, for which the resulting difference in the total supply cost between the centralized and iterative algorithms was $0.003 \%$. The simulation time was 87 seconds for the iterative algorithm and 39 hours for the centralized 445 algorithm. Thus, the simulation time does not increase as dramatically for the iterative algorithm in the presence of series FACTS devices as it does for the centralized one.

An additional advantage of the iterative approach is that even if the algorithm is stopped before it converges, the dispatch obtained on each iteration is feasible with the exception of the risk constraint. Thus, if the runtime is limited, once this limit has been reached, the algorithm can be stopped and the dispatch with the smallest risk constraint violation can be chosen among the completed iterations. This feature can be useful to system operators, for whom the timeliness of a solution may sometimes be more important than its quality.

Table 2: Comparison of Iterative and Centralized Solution, 24-bus system w/o FACTS

\begin{tabular}{|c|c|c|c|c|c|}
\hline \multirow{2}{*}{ Case } & \multirow{2}{*}{$\boldsymbol{N}_{\text {Cost, }} \mathbf{\%}$} & \multicolumn{2}{|c|}{ Time $_{\text {centr }}, \mathbf{s}$} & \multicolumn{2}{c|}{ Time $_{\text {iter }}, \mathbf{s}$} \\
\cline { 3 - 6 } & & flat & warm & flat & warm \\
\hline 1 & $1.5 \cdot 10^{-4}$ & 865.5 & 217.9 & 31.4 & 27.9 \\
2 & $4.0 \cdot 10^{-5}$ & 1555.4 & 179.9 & 14.3 & 14.7 \\
3 & $1.1 \cdot 10^{-6}$ & 1158.6 & 2088.6 & 14.2 & 14.8 \\
4 & $2.4 \cdot 10^{-5}$ & 1499.7 & 164.8 & 8.7 & 9.0 \\
5 & $2.8 \cdot 10^{-5}$ & 2075.6 & 616.7 & 11.6 & 11.6 \\
6 & $9.9 \cdot 10^{-5}$ & 1903.7 & 257.5 & 10.3 & 9.1 \\
7 & $5.2 \cdot 10^{-5}$ & 1752.4 & 218.5 & 18.7 & 19.1 \\
8 & $3.2 \cdot 10^{-5}$ & 6911.9 & 159.2 & 17.5 & 16.6 \\
9 & $1.3 \cdot 10^{-7}$ & 1842.8 & 149.7 & 6.8 & 6.5 \\
10 & $3.7 \cdot 10^{-5}$ & 2079.5 & 324.0 & 14.1 & 12.8 \\
\hline
\end{tabular}


In order to analyze the impact of series FACTS devices on system operation, the following steps were undertaken. First, for each considered configuration of the 118-bus system the most cost-efficient generation dispatch in the absence of security constraints was determined. Then, the minimum possible risk value corresponding to the obtained dispatch was calculated. If no power flow control devices are installed in the system, the risk of system operation is a fixed value for a given generation dispatch and no optimization is possible. Series FACTS devices can reroute line flows and reduce the risk without changing generator outputs except for the slack bus output, which has to be adjusted to account 465 for a change in active power losses. Figure 4 a shows the initial values of the risk for all system configurations. One can see that series FACTS devices helped reduce the risk of system operation by approximately $50 \%$.

For a better visualization of the impact of series FACTS devices on system operation, the minimum possible total supply cost was analyzed as a function of the desired risk level. To do this, the AC RCOPF problem was solved a number of times, each time setting a stricter risk limit until the minimum possible risk value for a given loading pattern was achieved. The resulting curves are presented in Fig. $4 \mathrm{~b}$ for the base case with and without corrective power flow control. Cutting this graph vertically, one can see the reduction in the supply cost for a certain risk level that series FACTS devices helped achieve. Similarly, a horizontal cut will show by how much these devices were able to reduce the total risk for a certain supply cost. It can be seen from this graph that corrective power flow control by means of series FACTS devices had significant positive effects on the efficiency and security of the considered system.

While series FACTS devices increase the flexibility of the power grid, they also add complexity to the problem of its optimal control. Therefore, it was important to understand how series FACTS devices would affect the speed of the proposed algorithm for solving the AC RCOPF problem. As in previous sections, the desired level of risk was set to $30 \%$ of the initial risk and the RCOPF problem was solved by the iterative algorithm. The resulting simulation times

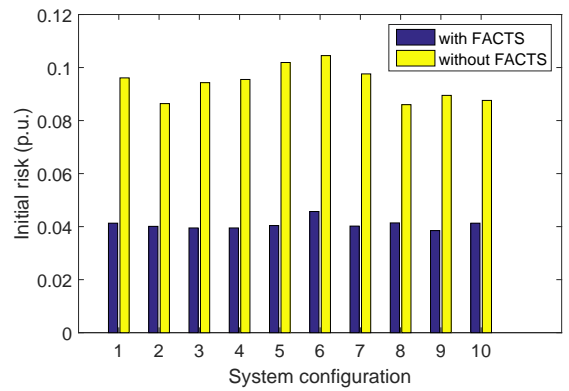

(a) Initial value of total risk

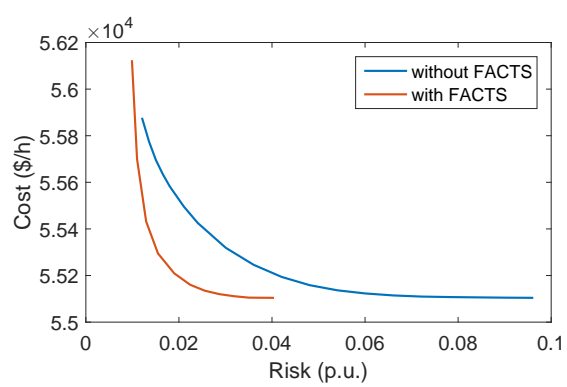

(b) Supply cost as a function of risk

Figure 4: Impact of series FACTS devices on system risk and cost 
for the systems with and without series FACTS devices are shown in Fig. 5 One can see that the proposed algorithm was on average four times slower for the systems with series FACTS devices compared to the case without FACTS devices. However, this increase was reasonably small considering the number of introduced control variables.

\subsection{Parallelization of Iterative Algorithm}

Parallelization of the proposed iterative algorithm, discussed in Sec. 6.3. can significantly reduce the simulation time, which is crucial for applying AC $\mathrm{RCOPF}$ in system operation. However, one cannot simply assume that the decrease in the computation time is linearly proportional to the number of available processors, because there exists communication overhead between the parallel workers and client. To determine the realistic behavior of the parallelized algorithm, a server with two $2.2 \mathrm{GHz}$ Intel Xeon CPUs was used. Since the parallelization occurs within one iteration of the proposed algorithm (see Fig. 2), all results were obtained for one iteration of the algorithm. The 24-bus system without series FACTS devices and 118-bus system with and without series FACTS devices were used and the number of parallel workers varied from 0 (no parallelization) to 16 (maximum available on the machine). In Fig. 6, the simulation time is shown as a function of the number of parallel workers.

Several conclusions can be drawn based on the presented results. First, the relation between the runtime and the number of parallel workers is nonlinear due to the communication overhead. Second, while in theory each N-1 contingency can be solved on a separate processor, in practice much fewer parallel processors are required to achieve the best performance. For instance, when more than 51010 processors were used for the 24-bus system, the communication overhead outweighed the advantage of solving optimization subproblems in parallel and the overall simulation time increased. Last, the larger the size of the power system, the more beneficial the parallelization becomes in terms of reducing the overall simulation time relative to the sequential solution. This is so because as the size of the system increases, the time to solve each optimization subproblem grows faster than the communication overhead.

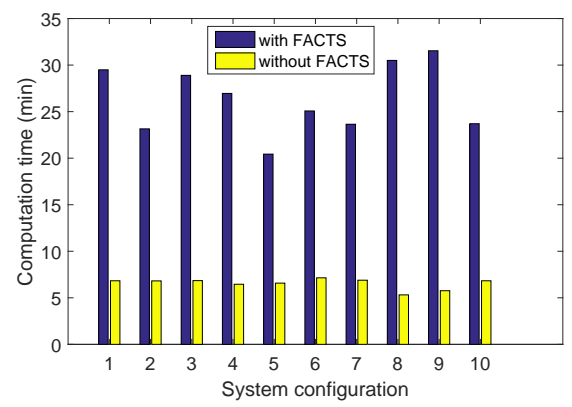

Figure 5: Computation time for iterative algorithm 


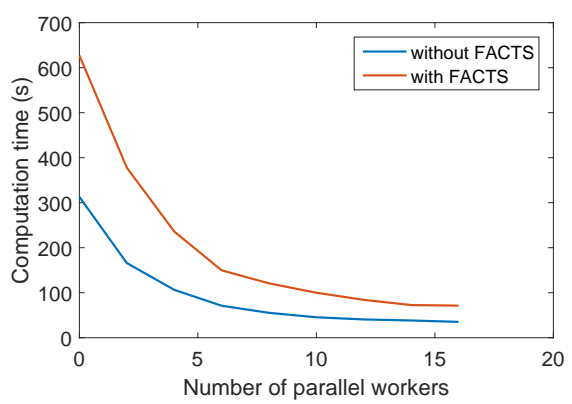

(a) 118-bus system

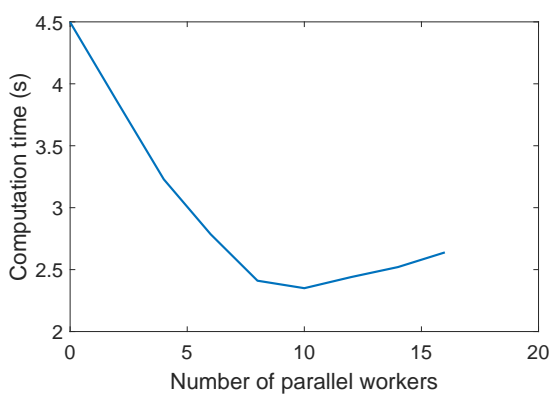

(b) 24-bus system without FACTS

Figure 6: Computation time for one iteration for different number of parallel workers

In general, the optimal number of parallel processors for solving the $\mathrm{AC}$ RCOPF problem by the proposed algorithm would depend on the power system under consideration and on the machine used. However, it is clear that parallelization is indispensable to obtaining a timely solution to the RCOPF problem for large-scale power systems.

\section{Conclusion}

This paper presents an iterative decomposed algorithm for solving the riskconstraint OPF problem. The problem is formulated for an AC model of a power system with corrective power flow control enabled by multiple series FACTS devices and includes outages of up to two elements as credible contingencies. The algorithm is based on so-called locational security impact factors, which represent the sensitivities of the total risk with respect to changes in generator outputs. The advantages of this algorithm over the brute-force centralized 530 approach include its lower computational complexity, ability to handle contingencies with no power flow solution, and possibility to perform a significant part of computations in parallel. The paper provides a parallelization scheme that enables the highest reduction in the simulation time.

The proposed algorithm has been tested on the 24-bus and 118-bus systems. The simulation results indicate that this algorithm converges to the same solution as the centralized one, which shows that it is able to handle a non-linear $\mathrm{AC}$ problem and additional non-linearities introduced by the presence of multiple series FACTS devices in the system. Furthermore, the iterative algorithm is substantially faster and less dependent on the starting point than the centralized approach. In addition, while the simulation time for the iterative algorithm increases when corrective power flow control is employed, this increase is reasonably small compared to the number of introduced control variables. The parallelization of the iterative algorithm helps reduce the simulation time, although in practice the optimal number of parallel processors is determined by communication overhead rather than the number of independent optimization 
subproblems. The simulation results also demonstrate that if multiple series FACTS devices are present in the system, its efficiency and security can be significantly improved.

Future extensions of this research might include considering generator outthe $\mathrm{AC}$ risk-constrained $\mathrm{OPF}$ problem and accelerate the computation without sacrificing the quality of the obtained solution.

\section{References}

[1] O. Alsac, B. Stott, Optimal load flow with steady-state security, IEEE Transactions on Power Apparatus and Systems (3) (1974) 745-751.

[2] A. Monticelli, M. V. F. Pereira, S. Granville, Security-constrained optimal power flow with post-contingency corrective rescheduling, Power Systems, IEEE Transactions on 2 (1) (1987) 175-180.

[3] F. Capitanescu, M. Glavic, D. Ernst, L. Wehenkel, Contingency Filtering Techniques for Preventive Security-Constrained Optimal Power Flow, IEEE Transactions on Power Systems 22 (4) (2007) 1690-1697. doi:10.1109/ TPWRS.2007.907528.

[4] J. McCalley, S. Asgarpoor, L. Bertling, R. Billinion, H. Chao, J. Chen, J. Endrenyi, R. Fletcher, A. Ford, C. Grigg, Probabilistic security assessment for power system operations, in: IEEE Power Engineering Society General Meeting, 2004, pp. 212-220.

[5] D. Kirschen, D. Jayaweera, D. Nedic, R. Allan, A Probabilistic Indicator of System Stress, IEEE Transactions on Power Systems 19 (3) (2004) 16501657. doi:10.1109/TPWRS.2004.831665.

[6] J. D. McCalley, V. Vittal, N. Abi-Samra, An overview of risk based security assessment, in: IEEE Power Engineering Society Summer Meeting, Vol. 1, 1999, pp. 173-178.

[7] D. Kirschen, D. Jayaweera, Comparison of risk-based and deterministic security assessments, IET Generation, Transmission \& Distribution 1 (4) (2007) 527. doi:10.1049/iet-gtd:20060368.

[8] Y. Dai, J. D. McCalley, N. Abi-Samra, V. Vittal, Annual risk assessment for overload security, IEEE Transactions on Power Systems 16 (4) (2001) 616-623.

[9] M. Ni, J. McCalley, V. Vittal, T. Tayyib, Online risk-based security assessment, IEEE Transactions on Power Systems 18 (1) (2003) 258-265. doi:10.1109/TPWRS.2002.807091.

[10] W. Fu, J. D. McCalley, Risk based optimal power flow, in: IEEE Power Tech Proceedings, 2001. 
[11] Y. Li, J. D. McCalley, Risk-based optimal power flow and system operation state, in: IEEE PES General Meeting, 2009.

[12] J. He, L. Cheng, D. Kirschen, Y. Sun, Optimising the balance between security and economy on a probabilistic basis, IET Generation, Transmission \& Distribution 4 (12) (2010) 1275-1287. doi:10.1049/iet-gtd.2010.0039

[13] L. Roald, M. Vrakopoulou, G. Andersson, F. Oldewurtel, Risk constrained optimal power flow with probabilistic guarantees, in: Power Systems Computation Conference (PSCC), 2014.

[14] Q. Wang, J. D. McCalley, T. Zheng, E. Litvinov, A Computational Strategy to Solve Preventive Risk-Based Security-Constrained OPF, IEEE Transactions on Power Systems 28 (2) (2013) 1666-1675. doi:10.1109/TPWRS. 11. 2012.2219080

[15] R. Yang, G. Hug, Potential and Efficient Computation of Corrective Power Flow Control in Cost vs. Risk Trade-Off, IEEE Transactions on Smart Grid 5 (4) (2014) 2033-2043. doi:10.1109/TSG.2014.2322616.

[16] E. Karangelos, P. Panciatici, L. Wehenkel, Whither probabilistic security management for real-time operation of power systems?, in: Bulk Power System Dynamics and Control - IX Optimization, Security and Control of the Emerging Power Grid (IREP), IREP Symposium, 2013, pp. 1-17. doi:10.1109/IREP.2013.6629408.

[17] F. Capitanescu, Enhanced risk-based SCOPF formulation balancing operation cost and expected voluntary load shedding, Electric Power Systems Research 128 (2015) 151-155. doi:10.1016/j.epsr.2015.07.007.

[18] C. E. Murillo-Snchez, R. D. Zimmerman, C. L. Anderson, R. J. Thomas, A stochastic, contingency-based security-constrained optimal power flow for the procurement of energy and distributed reserve, Decision Support Systems 56 (2013) 1-10. doi:10.1016/j.dss.2013.04.006.

[19] P. Etingov, A. Oudalov, N. Voropai, A. Germond, R. Cherkaoui, Coordinated emergency control of load shedding and FACTS devices, in: IEEE Power Tech, 2005.

[20] L. Goel, Y. Ding, P. Wang, Reliability assessment of restructured power systems using optimal load shedding technique, IET Generation, Transmission \& Distribution 3 (7) (2009) 628-640. doi:10.1049/iet-gtd.2008.0308.

[21] A. Oudalov, R. Cherkaoui, A. Germond, M. Emery, Coordinated power flow control by multiple FACTS devices, in: IEEE Power Tech, Bologna, 2003.

[22] W. Shao, V. Vittal, LP-Based OPF for Corrective FACTS Control to Relieve Overloads and Voltage Violations, IEEE Transactions on Power Systems 21 (4) (2006) 1832-1839. doi:10.1109/TPWRS.2006.881127. 
[23] N. G. Hingorani, L. Gyugyi, Understanding FACTS: Concepts and Technology of Flexible AC Transmission Systems, IEEE Press, 1999.

[24] R. Yang, G. Hug-Glanzmann, Regression-based corrective power flow control for system risk minimization, in: IEEE Power and Energy Society General Meeting (PES), 2013.

[25] F. Kreikebaum, D. Das, Y. Yang, F. Lambert, D. Divan, Smart Wires: A distributed, low-cost solution for controlling power flows and monitoring transmission lines, in: IEEE Innovative Smart Grid Technologies Conference Europe (ISGT Europe), 2010.

[26] F. Capitanescu, J. L. Martinez Ramos, P. Panciatici, D. Kirschen, A. Marano Marcolini, L. Platbrood, L. Wehenkel, State-of-the-art, challenges, and future trends in security constrained optimal power flow, Electric Power Systems Research 81 (8) (2011) 1731-1741. doi:10.1016/j. epsr.2011.04.003.

[27] F. Capitanescu, Critical review of recent advances and further developments needed in AC optimal power flow, Electric Power Systems Research 136 (2016) 57-68. doi:10.1016/j.epsr.2016.02.008

[28] R. Yang, G. Hug, Locational security impact factors for security index constrained economic dispatch problem, in: North American Power Symposium (NAPS), 2013, 2013. doi:10.1109/NAPS.2013.6666911.

[29] D. Shchetinin, G. Hug, Locational security impact factors for riskconstrained AC OPF, in: Innovative Smart Grid Technologies Conference North America (ISGT North America), 2015.

[30] C. Grigg et al., The IEEE reliability test system-1996, IEEE Transactions on Power Systems 14 (3) (1999) 1010-1020. 